\title{
Optimal Location and Control Parameter Settings of UPFC using Differential Evolution Algorithm
}

\author{
R.Vanitha \\ Research Scholar \\ Sathyabama University, Chennai, India
}

\author{
M.Sudhakaran \\ Associate Professor, Pondicherry Engineering \\ College, Pondicherry, India
}

\begin{abstract}
The main objective of this paper is to find the optimal location and control parameters settings of Unified Power Flow Controller (UPFC) with regard to power loss minimization. The proposed algorithm is based on steady state power injection model of UPFC. In this paper, two Evolutionary optimization techniques, namely Differential Evolution Algorithm (DE) and Genetic Algorithm (GA) are employed to solve optimal power flow problems. IEEE 14 bus \& IEEE 30 bus test power systems are used for studies. The obtained results indicate that both techniques can successfully find the optimal location and control parameter settings of UPFC, but DE is faster than GA from the time perspective.
\end{abstract}

\section{Keywords}

Optimal power flow, Power loss minimization, Genetic Algorithm, Differential Evolution, Evolutionary Optimization technique

\section{INTRODUCTION}

Optimal power flow (OPF) is a nonlinear programming problem (NLP) which is used to minimize a desired objective function subject to certain system constraints by determining the optimal control parameter settings. An optimization technique is used to determine the global optimum solution to a given OPF problem. Optimization means finding the best-suited solution for a problem within its given constraints and flexibilities.

Several conventional techniques like nonlinear programming (NLP), quadratic programming, mixed integer programming and Newton techniques are used to obtain the solution for optimal power flow problems [1, 2]. The limitations of these methods lead to the development of Evolutionary computing techniques like genetic algorithm (GA), simulated annealing (SA), particle swarm optimization (PSO), tabu search (TS), Differential Evolution algorithm (DE) and many more [ $3-11]$. These algorithms can solve complex optimization problems which are non-linear, non- continuous, non -differentiable and multi- dimensional.

Differential Evolution (DE) algorithm is a stochastic population based search optimization algorithm. It has certain advantages like finding the true global minimum irrespective of initial parameter values. It uses few control parameters to find the true global minimum. It is similar to Genetic algorithm which uses mutation, crossover, and selection operators. It uses the mutation operation as a search mechanism and selection operation as a direction mechanism. It employs a greedy selection process which leads to faster convergence compared with Genetic algorithm [12].

In transmission systems, Flexible AC transmission systems (FACTS) increase system transmission capacity and provide flexibility in power flow control. [13, 14]. These FACTS devices are used to overcome regulatory problems in transmission systems. Unified Power Flow Controller (UPFC) is the most sophisticated FACTS device. It can independently or simultaneously controls the active power, the reactive power and the bus voltage to which it is connected [15].

Several steady state models are available for UPFC to be implemented in power flow program based on Newton- Raphson algorithm. Some are decoupled UPFC model, injection UPFC model and Comprehensive NR UPFC model. Each model has its own merits and demerits. A mathematical model for UPFC which will be referred as UPFC injection model is used in this study [16]. The advantage of this method is that it can be easily implemented in existing power flow program and UPFC can be adjusted to work as series compensator, voltage regulator, or phase shifter [17]

In this paper, Differential Evolution algorithm is used in OPF technique to determine the optimal location and control parameter settings of UPFC for minimization of total real power loss in the power system.

This paper is structured as follows. In section 2, Basic concepts of UPFC are introduced. In section 3, the problem for the study is formulated. Section 4 presents an overview of Differential Evolution algorithm. Section 5 discusses the results for DE and GA algorithms and its comparisons. Section 6 summaries the main ideas presented in this paper.

\section{BASIC CONCEPTS OF UPFC}

\subsection{Operating Principle of UPFC}

The Unified Power Flow Controller consists of two voltage sourced converters connected back-to- back through a common DC link provided by a DC storage capacitor. (Fig 1).

The primary function of converter 1 is to supply or absorb the real power demanded by converter 2 through the common DC link. Converter 1 can also generate or absorb controllable reactive power if it is desired and thereby provides independent shunt reactive compensation for the line. The main function of UPFC is performed by converter 2 by injecting an AC voltage with controllable magnitude and phase angle in series with the transmission line via a series transformer. The required reactive 
power is supplied or absorbed locally by converter 2 and active power is exchanged as a result of the series injection voltage.

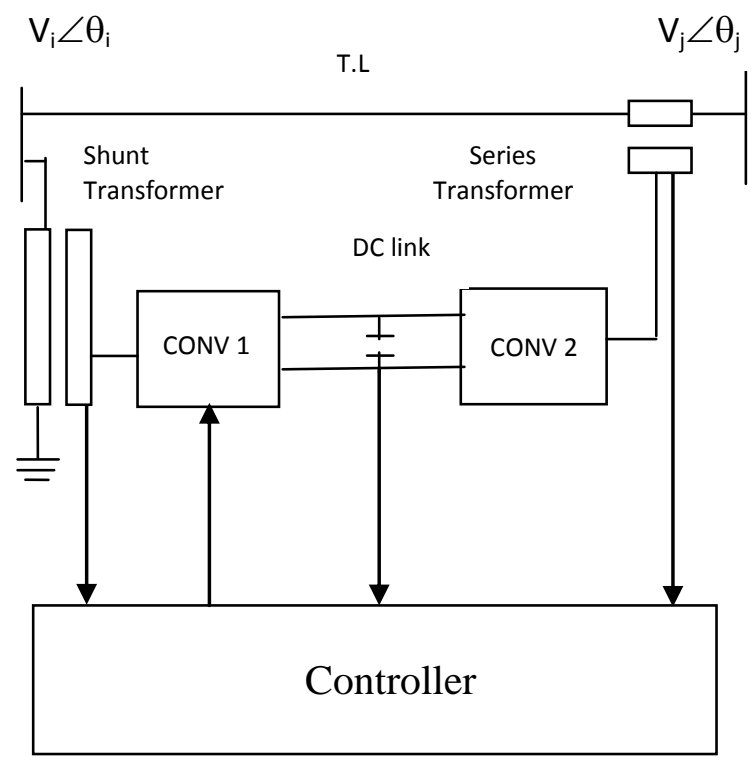

Fig 1 : UPFC Schematic Diagram

\subsection{UPFC Steady State Injection Model}

UPFC can be easily incorporated into the power flow equation using the steady state injection model [16] UPFC power injection model is presented in Fig. 2.

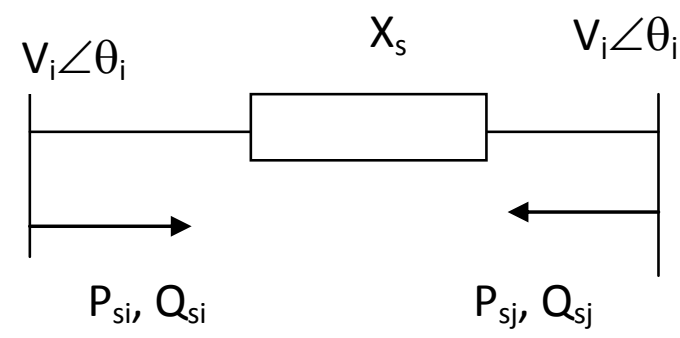

Fig 2: UPFC Model

$P_{s i}=r b_{s} V_{i} V_{j} \sin \left(\theta_{i j}+\gamma\right)$

$P_{s j}=-r b_{s} V_{i} V_{j} \sin \left(\theta_{i j}+\gamma\right)$

$Q_{s i}=r b_{s} V_{i}^{2} \cos \gamma$

$Q_{s j}=-r b_{s} V_{i} V_{j} \cos \left(\theta_{i j}+\gamma\right)$

If a UPFC is located between node $\mathrm{i}$ and node $\mathrm{j}$ in a power system, the admittance matrix is modified by adding a reactance equivalent to Xs between the nodes $\mathrm{i}$ and $\mathrm{j}$.The Jacobian matrix is modified by addition of appropriate powers.

\section{PROBLEM FORMULATION}

To achieve the best utilization of the existing transmission systems, UPFC device should be installed in such a place to minimize the total real power loss. In this paper the objective function chosen is minimization of total real power loss $\mathrm{P}_{\text {loss }}$ in power system under several loading conditions.

\subsection{Objective Function}

$$
\min P_{\text {loss }}=\sum_{i=1}^{n l} G_{i, j}\left\{V_{i}^{2}+V_{j}^{2}-2 V_{i} V_{j} \cos \left(\delta_{i}-\delta_{j}\right)\right\}
$$

Where

$$
\begin{array}{ll}
\text { Ploss } & : \text { Active power loss function } \\
\mathrm{G}_{\mathrm{ij}} & : \text { Conductance of line } \mathrm{i}-\mathrm{j} \\
\mathrm{V}_{\mathrm{i}} & : \text { Voltage magnitude at bus } \mathrm{i} \\
\mathrm{V}_{\mathrm{j}} & : \text { Voltage magnitude at bus } \mathrm{j} \\
\delta_{\mathrm{i}} & : \text { Voltage angle at bus } \mathrm{i} \\
\delta \mathrm{j} & : \text { Voltage angle at bus } \mathrm{j} \\
\mathrm{nl} & : \text { Total number of transmission lines }
\end{array}
$$

\subsection{System Constraints}

\subsubsection{Equality Constraint: Power flow equation}

$$
\begin{aligned}
& P_{G i}=P_{D i}+\sum_{j=1}^{n b}\left|V_{i}\right|\left|V_{j} \| Y_{i j}\right| \cos \left(\theta_{i j}-\delta_{i}+\delta_{j}\right) \\
& Q_{G i}=Q_{D i}+\sum_{j=1}^{n b}\left|V_{i}\right|\left|V_{j} \| Y_{i j}\right| \sin \left(\theta_{i j}-\delta_{i}+\delta_{j}\right)
\end{aligned}
$$

Where

$$
\begin{array}{ll}
\mathrm{P}_{\mathrm{Gi}} & : \text { real power generation at bus } \mathrm{i} \\
\mathrm{Q}_{\mathrm{Gi}} & : \text { reactive power generation at bus } \mathrm{i} \\
\mathrm{P}_{\mathrm{Di}} & : \text { real power demand at bus } \mathrm{i} \\
\mathrm{Q}_{\mathrm{Di}} & : \text { reactive power demand at bus } \mathrm{i} \\
\theta_{\mathrm{ij}} & : \text { angle of bus admittance element } \mathrm{i}, \mathrm{j} \\
\mathrm{Y}_{\mathrm{ij}} & : \text { magnitude of bus admittance element } \mathrm{i}, \mathrm{j} \\
\mathrm{nb} & : \text { Total number of buses }
\end{array}
$$

\subsubsection{Inequality Constraints}

$V_{i}^{\min } \leq V_{i} \leq V_{i}^{\max }$

$$
\begin{aligned}
& P_{G i}^{\min } \leq P_{G i} \leq P_{G i}^{\max } \\
& Q_{c i}^{\min } \leq Q_{c i} \leq Q_{c i}^{\max }
\end{aligned}
$$


$r_{\min } \leq r \leq r_{\max }$

$\gamma_{\min } \leq \gamma \leq \gamma_{\max }$

Where

$$
\begin{array}{ll}
\mathrm{V}_{\mathrm{i}}{ }^{\min }, \mathrm{V}_{\mathrm{i}}{ }^{\max }: \begin{array}{l}
\text { upper and lower limits of voltage } \\
\text { magnitude at bus } \mathrm{i}
\end{array} \\
\mathrm{P}_{\mathrm{Gi}}{ }^{\min }, \mathrm{P}_{\mathrm{Gi}}{ }^{\max }: & \begin{array}{l}
\text { upper and lower limits of power } \\
\text { generated by generator } \mathrm{i} .
\end{array} \\
\mathrm{Q}_{\mathrm{ci}}{ }^{\min }, \mathrm{Q}_{\mathrm{ci}}{ }^{\max }: & \begin{array}{l}
\text { upper and lower limits of reactive } \\
\text { power source i. }
\end{array} \\
\mathrm{r}, \gamma & : \text { UPFC parameters }
\end{array}
$$

\section{OVERVIEW OF DIFFERENTIAL EVOLUTION ALGORITHM}

In 1995, Storn and Price first proposed Differential Evolution (DE) algorithm [18]. DE is a new stochastic parallel direct search method used for Global Optimization. It has been widely studied and applied in many fields [19, 20, 21]. This algorithm uses a population $\mathrm{P}$ which consists of $\mathrm{N}$ individuals that evolve over $\mathrm{G}$ generations to reach an optimal solution. The number of the individual`s $\mathrm{N}$ in the population $\mathrm{P}$ remains constant during the minimization process. The dimension $\mathrm{D}$ of each individual will be equal to the number of decision or design parameters.

$$
\begin{aligned}
& P=\left[Y_{1}^{(G)}, \ldots \ldots \ldots \ldots \ldots Y_{N}^{(G)}\right] \\
& \left.Y_{i}^{G}=\left[X_{1 i}^{(G)}, \ldots \ldots \ldots \ldots . . X_{D i}^{(G)}\right]\right]^{T}
\end{aligned}
$$

Where

$$
\begin{array}{ll}
\mathrm{i} & : 1,2 \ldots \ldots \mathrm{N} \\
\mathrm{P} & : \text { Population Vector } \\
\mathrm{X} & : \text { decision Variable } \\
\mathrm{D} & : \text { number of decision variable } \\
\mathrm{G} & : \text { number of Generation } \\
\mathrm{N} & : \text { Population size }
\end{array}
$$

Like other evolutionary algorithms, the initial population is chosen randomly and uniformly over the entire parameter space. The optimization process is carried out using the three basic operations like Mutation, Cross over and Selection for all individuals until a stopping criterion is met.

\subsection{DE Algorithm}

Initialization

Evaluation

\section{Repeat}

Mutation

Crossover

Evaluation

Selection

Until (termination criteria are met)

\subsection{DE Optimization Process}

\subsubsection{Initialization}

The first step in Differential Evolution algorithm is to create an initial population. Each decision or design parameter of each individual of population is assigned with a random value and that value must lie within the feasible bounds of the decision variable.

$Y_{i, j}^{(0)}=Y_{j}^{\min }+\left(Y_{j}^{\max }-Y_{j}^{\min }\right) * \operatorname{rand}[0,1]$

Where

$$
\begin{aligned}
& \mathrm{i} \quad: \quad 1,2 \ldots \ldots \mathrm{N} \\
& \mathrm{j} \quad: \quad 1,2 \ldots \ldots . \mathrm{D} \\
& \mathrm{Y}_{\mathrm{j}}^{\text {min }}, \mathrm{Y}_{\mathrm{j}}^{\text {max }}: \text { Maximum and Minimum limit of } \mathrm{j}^{\text {th }} \\
& \text { decision Vector }
\end{aligned}
$$

\subsubsection{Mutation}

The mutation operator introduces new parameters into the population. For crossover and mutation several types of strategies are in use. The strategy which is implemented is explained in detail. The mutation operator derives the mutant vector $\mathrm{Y}_{\mathrm{M}}$ by using the weighted difference of two randomly selected population vectors $\mathrm{Y}_{\mathrm{b}}$ and $\mathrm{Y}_{\mathrm{c}}$ and adds to another randomly selected population vector $\mathrm{Y}_{\mathrm{a}}$. All of these randomly selected vectors must be different from each other. The convergence is improved by scaling the difference vector by a user defined constant known as the scaling constant $\mathrm{S}$ in the range $[0-1.2]$.

$Y_{M}^{(G)}=Y_{a}^{(G)}+S\left(Y_{b}^{(G)}-Y_{c}^{(G)}\right)$

Where

$$
\begin{aligned}
& \mathrm{Y}_{\mathrm{a}}, \mathrm{Y}_{\mathrm{b}}, \mathrm{Y}_{\mathrm{c}} \quad \begin{array}{l}
\text { randomly chosen vectors from the } \\
\text { population }
\end{array} \\
& \mathrm{Y}_{\mathrm{a}}, \# \mathrm{Y}_{\mathrm{b}} \# \mathrm{Y}_{\mathrm{c}} \\
& \mathrm{S}=\text { Scaling factor }[0-1.2]
\end{aligned}
$$

\subsubsection{Crossover}

The crossover operator creates the trial vectors, which are used in the selection process. The mutant vector is mixed with target (parent) vector to yield trial vector by creating a random number using any one of the distributions like uniform distribution, binomial distribution or exponential distribution and compared against a user defined constant referred to as the crossover constant $\mathrm{CR}$ in the range [0 - 1]. If the value of the random number is less or equal than the value of the crossover constant, the parameter will come from the mutant vector, otherwise the parameter comes from the parent vector.

$Y_{T}^{(G)}=\left\{\begin{array}{c}Y_{M}^{(G)} \text { if } \eta_{j} \leq \text { CRorj }=q \\ Y_{i, j}^{(G)}, \text { otherwise }\end{array}\right\}$ 
Where

$\mathrm{q} \quad$ : randomly chosen index from $\{1,2 \ldots \mathrm{D}\}$

$\mathrm{Y}_{\mathrm{i}, \mathrm{j}}{ }^{(\mathrm{G})}$ : target Vector or Parent Vector

$\mathrm{Y}_{\mathrm{M}}{ }^{(\mathrm{G})}$ : mutant vector

$\mathrm{Y}_{\mathrm{T}}^{(\mathrm{G})}$ : trial vector

CR : Crossover Constant [0 - 1]

\subsubsection{Selection}

The selection operator chooses the vectors that are going to be the population in next generation. Selection is the operation through which better offspring are generated. The fitness of the trial vector and the corresponding target vector is compared and better one will be selected by the operator.

$Y_{i, j}^{(G+1)}=\left\{\begin{array}{c}Y_{T}^{(G)} i f f\left(Y_{T}^{(G)}\right) \leq f\left(Y_{i, j}^{G}\right) \\ Y_{i, j}^{(G)}, \text { otherwise }\end{array}\right\}$

\section{DE IMPLEMENTATION RESULTS}

To verify the effectiveness and performance of the DE algorithm, IEEE 14 bus and IEEE 30 bus test power systems were used for test and compared with real coded Genetic algorithm [22, 23]. The algorithms are implemented in MATLAB 7.5 for different load conditions for determination of the optimal settings and location of UPFC to have minimum real power loss. Table $1 \& 2$ depicts the results of UPFC location, its optimal settings and total real and reactive power loss for IEEE 14 bus test system in DE \& GA. Table 3 shows the comparison results of DE and GA in terms of real power loss, execution time in sec. Table $4 \& 5$ reveals the results of IEEE 30 bus test system in DE \& GA and Table 6 compares the results of DE \& GA. All the studies are carried out on a PC with Intel core i5 $2.53 \mathrm{GHZ}$ processor and 4GB RAM.

Optimal Parameter Settings

$$
\begin{array}{ll}
\text { No. of Decision Variables } & : 2 \\
\text { Population size (N) } & : 30 \\
\text { Maximum Number of generations (G) } & : 50 \\
\text { Crossover Constant (CR) } & : 0.9 \\
\text { Scaling Factor (S) } & : 0.5
\end{array}
$$

\begin{tabular}{|c|c|c|c|c|}
\hline Cases & & $\begin{array}{c}\text { Status 1 } \\
\text { (Without } \\
\text { UPFC) }\end{array}$ & $\begin{array}{c}\text { Status 2 } \\
\text { ( With one UPFC) }\end{array}$ & $\begin{array}{c}\text { Status 3 } \\
\text { (With Two UPFC's) }\end{array}$ \\
\hline Normal loading & $\begin{array}{c}\Sigma \text { Ploss(MW) } \\
\Sigma \text { Qloss(MVAR) } \\
\text { Location (Bus No - Bus No) } \\
\text { UPFC Settings }\end{array}$ & $\begin{array}{c}13.3931 \\
26.2597 \\
- \\
-\end{array}$ & $\begin{array}{c}13.3119 \\
26.3271 \\
2-4 \\
\mathrm{r}=0.067, \gamma=157.48^{\circ}\end{array}$ & $\begin{array}{c}13.2983 \\
26.5089 \\
2-4 \& 2-5 \\
\mathrm{r}=0.039, \gamma=338.84^{\circ}\end{array}$ \\
\hline $\begin{array}{c}\text { Twice normal } \\
\text { loading }\end{array}$ & $\begin{array}{c}\Sigma \text { Ploss(MW) } \\
\Sigma \text { Qloss(MVAR) } \\
\text { Location (Bus No - Bus No) } \\
\text { UPFC Settings }\end{array}$ & $\begin{array}{c}70.859 \\
256.378 \\
- \\
-\end{array}$ & $\begin{array}{c}70.669 \\
256.32 \\
3-4 \\
\mathrm{r}=0.04, \gamma=201.28^{\circ}\end{array}$ & $\begin{array}{c}70.581 \\
257.71 \\
2-4 \& 3-4 \\
\mathrm{r}=0.035, \gamma=268.50^{\circ}\end{array}$ \\
\hline $\begin{array}{l}3 \text { Times normal } \\
\text { loading }\end{array}$ & $\begin{array}{c}\Sigma \text { Ploss(MW) } \\
\Sigma \text { Qloss(MVAR) } \\
\text { Location (Bus No - Bus No) } \\
\text { UPFC Settings }\end{array}$ & $\begin{array}{c}206.598 \\
789.599 \\
- \\
-\end{array}$ & $\begin{array}{c}205.93 \\
789.49 \\
3-4 \\
\mathrm{r}=0.035, \gamma=244.78^{\circ}\end{array}$ & $\begin{array}{c}205.87 \\
789.38 \\
3-4 \& 10-11 \\
\mathrm{r}=0.022, \gamma=47.80^{\circ}\end{array}$ \\
\hline $\begin{array}{c}\text { Active load } \\
\text { increasing thrice at } \\
\text { bus \#4 }\end{array}$ & $\begin{array}{c}\Sigma \text { Ploss(MW) } \\
\Sigma \text { Qloss(MVAR) } \\
\text { Location (Bus No - Bus No) } \\
\text { UPFC Settings }\end{array}$ & $\begin{array}{l}27.7635 \\
77.4348 \\
- \\
-\end{array}$ & $\begin{array}{c}27.5955 \\
77.8550 \\
2-4 \\
\mathrm{r}=0.06, \gamma=217.82^{\circ}\end{array}$ & $\begin{array}{c}27.5302 \\
78.128 \\
2-4 \& 2-5 \\
\mathrm{r}=0.038, \gamma=14.11^{\circ}\end{array}$ \\
\hline $\begin{array}{l}\text { Active \& Reactive } \\
\text { load increasing } \\
\text { thrice at bus \#9 }\end{array}$ & $\begin{array}{c}\Sigma \text { Ploss(MW) } \\
\Sigma \text { Qloss(MVAR) } \\
\text { Location (Bus No - Bus No) } \\
\text { UPFC Settings }\end{array}$ & $\begin{array}{l}22.7632 \\
77.6845\end{array}$ & $\begin{array}{c}22.6952 \\
77.4250 \\
5-6 \\
\mathrm{r}=0.028, \gamma=167.67^{\circ}\end{array}$ & $\begin{array}{c}22.666 \\
71.956 \\
2-4 \& 5-6 \\
\mathrm{r}=0.028, \gamma=167.67^{\circ}\end{array}$ \\
\hline
\end{tabular}

Table 1: Results of IEEE 14 -Bus System (Differential Evolution)

Table 2: Results of IEEE 14 -Bus System (Genetic Algorithm)

\begin{tabular}{|c|c|c|c|c|}
\hline Cases & & $\begin{array}{c}\text { Status 1 } \\
\text { (Without } \\
\text { UPFC) }\end{array}$ & $\begin{array}{c}\text { Status 2 } \\
\text { (With one UPFC) }\end{array}$ & $\begin{array}{c}\text { Status 3 } \\
\text { (With Two UPFC's) }\end{array}$ \\
\hline Normal loading & EPloss(MW) & 13.3931 & 13.3130 & 13.2989 \\
& LQloss(MVAR) & 26.2597 & 26.3312 & 26.5117 \\
& Location (Bus No-Bus No) & - & $2-4$ & $2-4 \& 2-5$ \\
& UPFC Settings & - & $\mathrm{r}=0.0667, \gamma=158.26^{\circ}$ & $\mathrm{r}=0.0389, \gamma=340.77^{\circ}$ \\
\hline
\end{tabular}




\begin{tabular}{|c|c|c|c|c|}
\hline $\begin{array}{l}\text { Twice normal } \\
\text { loading }\end{array}$ & $\begin{array}{c}\Sigma \text { Ploss(MW) } \\
\Sigma \text { Qloss(MVAR) } \\
\text { Location (Bus No - Bus No) } \\
\text { UPFC Settings }\end{array}$ & $\begin{array}{c}70.859 \\
256.378 \\
- \\
-\end{array}$ & $\begin{array}{c}70.672 \\
256.33 \\
3-4 \\
\mathrm{r}=0.0395, \gamma=219.01^{\circ}\end{array}$ & $\begin{array}{c}70.583 \\
257.72 \\
2-4 \& 3-4 \\
\mathrm{r}=0.0333, \gamma=263.79^{\circ}\end{array}$ \\
\hline $\begin{array}{c}3 \text { Times normal } \\
\text { loading }\end{array}$ & $\begin{array}{c}\Sigma P \operatorname{loss}(\mathrm{MW}) \\
\Sigma \mathrm{Q} \operatorname{loss}(\mathrm{MVAR}) \\
\text { Location (Bus No - Bus No) } \\
\text { UPFC Settings }\end{array}$ & $\begin{array}{c}206.598 \\
789.599 \\
- \\
-\end{array}$ & $\begin{array}{c}205.94 \\
789.54 \\
3-4 \\
r=0.0341, \gamma=225.52^{\circ}\end{array}$ & $\begin{array}{c}205.87 \\
789.33 \\
3-4 \& 10-11 \\
r=0.022, \gamma=44.42^{\circ}\end{array}$ \\
\hline $\begin{array}{c}\text { Active load } \\
\text { increasing thrice at } \\
\text { bus \#4 }\end{array}$ & $\begin{array}{c}\Sigma \text { Ploss(MW) } \\
\Sigma \text { Qloss(MVAR) } \\
\text { Location (Bus No - Bus No) } \\
\text { UPFC Settings }\end{array}$ & $\begin{array}{l}27.7635 \\
77.4348 \\
- \\
-\end{array}$ & $\begin{array}{c}27.5970 \\
77.5099 \\
2-4 \\
\mathrm{r}=0.058, \gamma=173.15^{\circ}\end{array}$ & $\begin{array}{c}27.5302 \\
78.1281 \\
2-4 \& 2-5 \\
\mathrm{r}=0.0378, \gamma=12.669^{\circ}\end{array}$ \\
\hline $\begin{array}{l}\text { Active \& Reactive } \\
\text { load increasing } \\
\text { thrice at bus \#9 }\end{array}$ & $\begin{array}{c}\Sigma \text { Ploss(MW) } \\
\Sigma \text { Qloss(MVAR) } \\
\text { Location (Bus No - Bus No) } \\
\text { UPFC Settings }\end{array}$ & $\begin{array}{l}22.7632 \\
77.6845\end{array}$ & $\begin{array}{c}22.6958 \\
77.4034 \\
5-6 \\
r=0.0271, \gamma=166.60^{\circ}\end{array}$ & $\begin{array}{c}22.6662 \\
77.9666 \\
2-4 \& 5-6 \\
r=0.0276, \gamma=357.30^{\circ}\end{array}$ \\
\hline
\end{tabular}

Table 3: Comparison between Differential Evolution Algorithm \& Genetic Algorithm (IEEE 14bus)

\begin{tabular}{|c|c|c|c|c|c|c|}
\hline \multirow[t]{2}{*}{ Case } & \multicolumn{2}{|c|}{$\begin{array}{c}\text { Decrease in real power loss } \Delta \\
\text { PL }(\mathrm{KW}) \\
\text { one UPFC }\end{array}$} & \multicolumn{2}{|c|}{$\begin{array}{c}\text { Decrease in real power } \\
\text { loss } \Delta \text { PL (KW) } \\
\text { Two UPFC's }\end{array}$} & \multicolumn{2}{|c|}{$\begin{array}{c}\text { CPU Time (sec) } \\
\text { Two UPFC's }\end{array}$} \\
\hline & DE & GA & DE & GA & DE & GA \\
\hline Normal loading & 81.2 & 80.1 & 94.8 & 94.2 & 3.73 & 22.2 \\
\hline Twice normal loading & 191 & 187 & 278 & 276 & 5.01 & 51.4 \\
\hline 3 Times normal loading & 668 & 658 & 728 & 728 & 4.84 & 50.6 \\
\hline Active load increasing thrice at bus \#4 & 168 & 167 & 233.3 & 202.3 & 5.49 & 51.4 \\
\hline $\begin{array}{c}\text { Active \& Reactive load increasing thrice at } \\
\text { bus \#9 }\end{array}$ & 68 & 67.4 & 97.2 & 97 & 5.05 & 55.7 \\
\hline
\end{tabular}

Table 4: Results of IEEE 30 -Bus System (Differential Evolution)

\begin{tabular}{|c|c|c|c|c|}
\hline Cases & & $\begin{array}{c}\text { Status 1 } \\
\text { (Without } \\
\text { UPFC) } \\
\end{array}$ & $\begin{array}{c}\text { Status } 2 \\
\text { ( With one UPFC) }\end{array}$ & $\begin{array}{c}\text { Status } 3 \\
\text { (With Two UPFC's) }\end{array}$ \\
\hline Normal loading & $\begin{array}{c}\Sigma \text { Ploss(MW) } \\
\Sigma \text { Qloss(MVAR) } \\
\text { Location (Bus No - Bus No) } \\
\text { UPFC Settings }\end{array}$ & $\begin{array}{c}17.599 \\
22.244 \\
- \\
-\end{array}$ & $\begin{array}{c}17.5223 \\
22.4053 \\
2-6 \\
\mathrm{r}=0.0658, \gamma=125.58^{\circ}\end{array}$ & $\begin{array}{c}17.5046 \\
22.6098 \\
2-4 \& 2-6 \\
\mathrm{r}=0.038, \gamma=309.18^{\circ}\end{array}$ \\
\hline $\begin{array}{l}\text { Twice normal } \\
\text { loading }\end{array}$ & $\begin{array}{c}\Sigma \text { Ploss(MW) } \\
\Sigma \text { Qloss(MVAR) } \\
\text { Location (Bus No - Bus No) } \\
\text { UPFC Settings }\end{array}$ & $\begin{array}{l}95.891 \\
333.77 \\
- \\
-\end{array}$ & $\begin{array}{c}95.689 \\
333.98 \\
6-7 \\
\mathrm{r}=0.061, \gamma=87.02^{\circ}\end{array}$ & $\begin{array}{c}95.501 \\
335.45 \\
2-6 \& 6-7 \\
\mathrm{r}=0.052, \gamma=305.21^{\circ}\end{array}$ \\
\hline $\begin{array}{c}\text { Active load } \\
\text { increasing thrice at } \\
\text { bus \#7 }\end{array}$ & $\begin{array}{c}\Sigma \text { Ploss(MW) } \\
\Sigma \text { Qloss(MVAR) } \\
\text { Location (Bus No - Bus No) } \\
\text { UPFC Settings }\end{array}$ & $\begin{array}{c}25.5924 \\
50.956 \\
- \\
-\end{array}$ & $\begin{array}{c}25.4839 \\
51.2496 \\
2-6 \\
\mathrm{r}=0.065, \gamma=138.78^{\circ}\end{array}$ & $\begin{array}{c}25.4680 \\
51.2363 \\
2-6 \& 6-7 \\
\mathrm{r}=0.053, \gamma=335.73^{\circ}\end{array}$ \\
\hline
\end{tabular}




\begin{tabular}{|c|c|c|c|c|}
\hline Active \& Reactive & SPloss(MW) & 24.0965 & 23.9970 & 23.9858 \\
load increasing & Qloss(MVAR) & 52.5682 & 52.8299 & 53.1688 \\
thrice at bus \#21 & Location (Bus No-Bus No) & - & $2-6$ & $2-4 \& 2-6$ \\
& UPFC Settings & - & $r=0.0608, \gamma=194.61^{\circ}$ & $r=0.0342, \gamma=18.43^{\circ}$ \\
& & & & \\
\end{tabular}

Table 5: Results of IEEE 30 -Bus System （Genetic Algorithm)

\begin{tabular}{|c|c|c|c|c|}
\hline Cases & & $\begin{array}{c}\text { Status 1 } \\
\text { (Without } \\
\text { UPFC) } \\
\end{array}$ & $\begin{array}{c}\text { Status } 2 \\
\text { ( With one UPFC) }\end{array}$ & $\begin{array}{c}\text { Status 3 } \\
\text { (With Two UPFC's) }\end{array}$ \\
\hline Normal loading & $\begin{array}{c}\Sigma \text { Ploss(MW) } \\
\Sigma \text { Qloss(MVAR) } \\
\text { Location (Bus No - Bus No) } \\
\text { UPFC Settings }\end{array}$ & $\begin{array}{c}17.599 \\
22.244 \\
- \\
-\end{array}$ & $\begin{array}{c}17.5239 \\
22.41 \\
2-6 \\
\mathrm{r}=0.0658, \gamma=125.58^{\circ}\end{array}$ & $\begin{array}{c}17.5056 \\
22.6137 \\
2-4 \& 2-6 \\
\mathrm{r}=0.0374, \gamma=309.22^{\circ}\end{array}$ \\
\hline $\begin{array}{l}\text { Twice normal } \\
\text { loading }\end{array}$ & $\begin{array}{c}\Sigma \text { Ploss(MW) } \\
\Sigma \text { Qloss(MVAR) } \\
\text { Location (Bus No - Bus No) } \\
\text { UPFC Settings }\end{array}$ & $\begin{array}{c}95.891 \\
333.77 \\
- \\
-\end{array}$ & $\begin{array}{c}95.693 \\
333.99 \\
6-7 \\
\mathrm{r}=0.0608, \gamma=84.33^{\circ}\end{array}$ & $\begin{array}{c}95.506 \\
335.47 \\
2-6 \& 6-7 \\
\mathrm{r}=0.0522, \gamma=304.92^{\circ}\end{array}$ \\
\hline $\begin{array}{c}\text { Active load } \\
\text { increasing thrice at } \\
\text { bus } \# 7\end{array}$ & $\begin{array}{c}\Sigma \text { Ploss(MW) } \\
\Sigma \text { Qloss(MVAR) } \\
\text { Location (Bus No - Bus No) } \\
\text { UPFC Settings }\end{array}$ & $\begin{array}{c}25.5924 \\
50.956 \\
- \\
-\end{array}$ & $\begin{array}{c}25.4970 \\
50.7654 \\
2-6 \\
\mathrm{r}=0.0533, \gamma=168.72^{\circ}\end{array}$ & $\begin{array}{c}25.4715 \\
51.2570 \\
2-6 \& 6-7 \\
\mathrm{r}=0.0466, \gamma=44.34^{\circ}\end{array}$ \\
\hline $\begin{array}{l}\text { Active \& Reactive } \\
\text { load increasing } \\
\text { thrice at bus } \# 21\end{array}$ & $\begin{array}{c}\Sigma \text { Ploss(MW) } \\
\Sigma \text { Qloss(MVAR) } \\
\text { Location (Bus No - Bus No) } \\
\text { UPFC Settings }\end{array}$ & $\begin{array}{c}24.0965 \\
52.5682 \\
- \\
-\end{array}$ & $\begin{array}{c}24.0053 \\
52.8601 \\
2-6 \\
\mathrm{r}=0.0641, \gamma=200.06^{\circ}\end{array}$ & $\begin{array}{c}23.9862 \\
53.1705 \\
2-4 \& 2-6 \\
\mathrm{r}=0.0351, \gamma=19.20^{\circ}\end{array}$ \\
\hline
\end{tabular}

Table 6: Comparison between Differential Evolution Algorithm \& Genetic Algorithm (IEEE 30bus)

\begin{tabular}{|c|c|c|c|c|c|c|}
\hline \multirow[t]{2}{*}{ Case } & \multicolumn{2}{|c|}{$\begin{array}{c}\text { Decrease in real power loss } \Delta \\
\text { PL }(\mathrm{KW}) \\
\text { one UPFC }\end{array}$} & \multicolumn{2}{|c|}{$\begin{array}{c}\text { Decrease in real power } \\
\text { loss } \Delta \text { PL (KW) } \\
\text { Two UPFC's }\end{array}$} & \multicolumn{2}{|c|}{$\begin{array}{c}\text { CPU Time (sec) } \\
\text { Two UPFC's }\end{array}$} \\
\hline & DE & GA & DE & GA & DE & GA \\
\hline Normal loading & 76.7 & 76.7 & 94.4 & 93.4 & 10.9 & 100.4 \\
\hline Twice normal loading & 202 & 202 & 390 & 385 & 13.7 & 193.6 \\
\hline Active load increasing thrice at bus \#7 & 108.5 & 108.5 & 124.4 & 120.9 & 12.7 & 126.2 \\
\hline $\begin{array}{l}\text { Active \& Reactive load increasing thrice at } \\
\text { bus \#21 }\end{array}$ & 99.5 & 99.5 & 110.7 & 110.3 & 14.2 & 119.8 \\
\hline
\end{tabular}

\section{CONCLUSION}

Optimal installation of FACTS device is required in achieving minimal total real power loss under different loading conditions. This paper attempted to find out the optimal location and parameters for one or two UPFC device(s) to minimize total real power loss using DE and GA techniques.

From the results it is found that in DE based optimal power flow method, the reduction of real power loss remains the same or better when compared with GA. When comparing CPU time 
execution, the convergence speed of DE is faster than GA and this is due to the fact that DE uses a non uniform crossover and employs a greedy selection process.

Therefore, the DE algorithm seems to be a promising approach for engineering optimization problems. With the above proposed algorithms, it is possible to locate the UPFC's in the transmission line such that proper power planning and operation can be achieved with minimum system losses.

\section{REFERENCES}

[1] Lee, K .Y. Park, Y. M., and Ortiz, J. L. 1985, "A United Approach to Optimal Real and Reactive Power Dispatch", IEEE Transactions on Power Apparatus and Systems, Vol. 104, pp. 1147-1153.

[2] Momoh, J. A., El-Hawary, E., and Adapa, R. February 1999 ,"A Review of Selected Optimal Power Flow Literature to 1993 Part I \& II", IEEE Transactions on Power Systems, Vol. 14, No. 1, , pp. 96-111.

[3] Malakar, T., Sinha, N., Goswami, S. K., and Saikia, L.C. 2010 "Optimal location and size determination of FACTS devices by using multiobjective optimal power flow", TENCON 2010 - IEEE Region 10 conference, pp. 474 478.

[4] Rahimzadeh,S., Tavakoli Bina, M., and Viki, A.H. 2010, "Simultaneous application of multi-type FACTS devices to the restructured environment: achieving both optimal number and location," IET Generation, Transmission and Distribution, Vol.4, pp. 349 - 362.

[5] Sen, S., Roy, P., Sengupta, S., and Chakrabart, A. 2010, "Genetic Algorithm based transmission loss optimization under deregulated environment", Joint international conference on Power Electronics, Drives and Energy systems, pp. 1-5.

[6] Khatibzadeh, A.A., Khanbeigi, G.A., Bamdadian, M.M., Naderi, H., and Sheikh-el-Eslami, M.K. 2011, " An improved Tabu search algorithm and PSO for unit commitment problem solving", 19th Iranian Conference on Electrical Engineering (ICEE), pp. 1

[7] Leeton, U., Uthitsunthorn, D., Kwannetr, U., Sinsuphun N., and Kulworawanichpong, T. 2010, "power Loss Minimization Using Optimal Power Flow Based on Particle Swarm Optimization", ECTI Conference, pp.469- 473.

[8] Shaheen, H.I., Rashed,G.I., and Cheng, S. J. 2007, "Application of Evolutionary Optimization Techniques for Optimal Location and Parameters Setting of Multiple UPFC Devices", Third international conference on natural computation, pp. $688-697$.

[9] Abdelsalam, H. A., Aly, G. A. M., Abdelkrim, M. K., and Shebl, M. 2004 " Optimal location of the Unified Power Flow Controller in Electrical power systems", IEEE Power systems conference and Exposition, , pp. 1391- 1396.
[10] Tripathy, S. Mishra, Lai, L.L., and Zhang, Q.P. 2006 , "Transmission Loss Reduction Based on FACTS and Bacteria Foraging Algorithm" PPSN IX - Lecturer notes in computer science, Vol. 4193, pp.222-231.

[11] Duvvuru, N., and Swarup, K.S. 2011, "A Hybrid Interior Point Assisted Differential Evolution Algorithm for Economic Dispatch", IEEE Transactions on Power Systems, Vol. 26, No.2, pp. $541-549$.

[12] Dervis Karaboga, and Selcuk Okdem, 2004, "A Simple and Global Optimization Algorithm for Engineering Problems: Differential Evolution Algorithm", Turk J Elec. Engin., Vol. 12, No.1, pp. 53-60.

[13] Hingorani,n,G., and Gyugyi, L., 1999 , "Understanding FACTS", The Institution of Electrical and Electronics Engineers.

[14] Mohan Mathur, R., and Rajiv K.Varma, 2002 , "Thyrisor Based FACTS Controllers for Electrical Transmission Systems", IEEE Press Series on Power Engineering, John Willy \& Sons, Inc. Publication, New York.

[15] Gyugyi, L., Schauder, C.D., William, S.L., Rietman, T.R., Torgerson, D.R., and Edris, A. October 1999, "The Unified Power Flow Controller: A New Approach to Power Transmission Control", IEEE Trans. On Power Delivery, Vol.14, No.4, pp 1374-1381.

[16] Noroozian, M., Angquist, L., Ghandhari, M., and Anderson, G. October 1997, "Use of UPFC for Optimal Power Flow Control", IEEE Trans. On Power Delivery, Vol.12, No.4, pp.1629-1634.

[17] EL-Sadek, M.Z., Ahmed, A., and Zidan, H.E. 2006, “ Comparision of Unified power flow Controller Models for Power Flow Studies", The Eleventh International Middle East Power Systems Conference (MEPCON'2006).

[18] Storn, R., and Price, K. 1997, "Differential evolution - a simple and efficient heuristic for global optimization over continuous spaces", J. Global Optim., Vol.11, pp. 341-359.

[19] Swagatam Das, and Ponnuthurai Nagaratnam Suganthan, FEB 2011, "Differential Evolution: A Survey of the Stateof-the-Art", IEEE Transactions on Evolutionary Computation, VOL. 15, NO. 1, pp.4-31.

[20] Varadarajan, M., and Swarup, K.S. 2008, "Differential evolutionary algorithm for optimal reactive power dispatch", International journal for electrical power and energy systems, Vol. 30, No. 8pages 435-441.

[21] Abou, A.A. , El Ela, Abido, M.A. , Spea, S.R. 2010, "Optimal power flow using differential evolution algorithm", Electric power system Research, Vol. 18, No. 7, pp. $878-885$.

[22] Pai, M.A., 2005, "Computer Techniques in power system Analysis".

[23] Hadi saadat, 2002, "Power System Analysis and Design". 